\title{
An evanescent approach for mitochondrial function assay of living cells
}

\author{
De-Ming Yang, $\mathrm{PhD},{ }^{\mathrm{a}, \mathrm{b}, *}$ Chien-Chang Huang, ${ }^{\mathrm{c}}$ Lung-Sen Kao, $\mathrm{PhD}$, ${ }^{\mathrm{c}, \mathrm{d}, \mathrm{e}}$ \\ Chung-Chih Lin, PhD, ${ }^{\mathrm{d}}$ Chin-Wen Chi, PhD ${ }^{\mathrm{a}, \mathrm{f}}$ Hsia Yu Lin, ${ }^{\mathrm{g}}$ Din Ping Tsai, PhD, ${ }^{\mathrm{g}}$ \\ Chen-Hsen Lee, $\mathrm{MD},{ }^{\mathrm{h}}$ Arthur Chiou, $\mathrm{PhD}^{\mathrm{b}}$ \\ ${ }^{a}$ Department of Medical Research and Education, Taipei Veterans General Hospital, Taipei, Taiwan \\ ${ }^{\mathrm{b}}$ Institute of Biophotonics Engineering, National Yang-Ming University, Taipei, Taiwan \\ ${ }^{\mathrm{c}}$ Institute of Biochemistry, National Yang-Ming University, Taipei, Taiwan \\ ${ }^{\mathrm{d}}$ Department of Life Sciences, National Yang-Ming University, Taipei, Taiwan \\ ${ }^{\mathrm{e}}$ Brain Research Center, University System of Taiwan, Taipei, Taiwan \\ ${ }^{\mathrm{f}}$ Institute of Pharmacology, National Yang-Ming University, Taipei, Taiwan \\ ${ }^{\mathrm{g}}$ Department of Physics, National Taiwan University, Taipei, Taiwan \\ ${ }^{\mathrm{h}}$ Department of Surgery, Taipei Veterans General Hospital, Taipei, Taiwan \\ Received 18 August 2005; accepted 10 October 2005
}

\begin{abstract}
Mitochondria are now known to function physiologically not only in the production of ATP as the major cellular energy source, but also in the regulation of intracellular signaling, in, for example, stress-induced apoptosis and buffering of cytosolic calcium. It should be noted, when interpreting mitochondrial studies in situ, that mitochondria within cells show heterogeneity in both function and location. We applied both conventional epifluorescence microscopy (EPIFM) and total-internalreflection fluorescence microscopy (TIRFM) in this study. Image data taken from TIRFM are excellent and markedly different from those taken from EPIFM. We further investigated the physiological variations of mitochondrial functions using an EPIFM/TIRFM dual-imaging system. This system permits further analysis of functions of mitochondria and other organelles with more precision than is possible using a traditional platform.
\end{abstract}

(C) 2005 Published by Elsevier Inc.

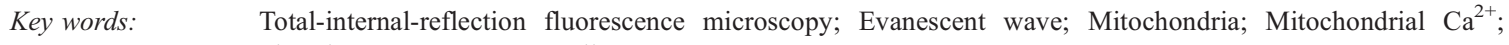
Pheochromocytoma PC12 cells

Previous approaches to the study of mitochondrial morphology and/or functions have included in situ microscopy and several methods to investigate isolated mitochondria, such as flow cytometry, capillary electrophoresis, patch clamping, and optical trapping (see review in [1]). Powerful microscopic and microanalytic techniques, such as electron microscopy (EM) and EM tomography, fluorescence mi-

No conflict of interest was reported by the authors of this paper.

This research is supported by grants from the National Science Council (NSC-90-2311-B-040-006), Taipei Veterans General Hospital (VGH94384), and Ministry of Education (Program for Promoting Academic Excellence of Universities, Grant 89-6-FA22-4).

* Corresponding author. Department of Medical Research and Education, Taipei Veterans General Hospital, No. 201 Sec. 2, Shih-Pai Road, 11217 Taipei, Taiwan.

E-mail address: dmyang@vghtpe.gov.tw (D.-M. Yang). croscopy, single- and two-photon confocal microscopy, and $\mathrm{X}$-ray microanalysis, have been applied in this research area but still have some limitations. Two major reasons for these limitations are: mitochondrial heterogeneity and diversity among tissues, organisms, and species with different physiological status [2,3]. For studying behavior, localization, and content measurement of mitochondria under optical microscopy or EM, fluorescent labeling and immunocytochemistry have been widely used for several decades. It is now known that mitochondria are organelles with many functions related to the dynamics of morphology [4], for example, generation of ATP, control of cell apoptosis [5], and buffering of cytosolic $\mathrm{Ca}^{2+}\left(\left[\mathrm{Ca}^{2+}\right]_{\mathrm{i}}\right)$ [6]. Spatial localization of mitochondria near the endoplasmic reticulum or the plasma membrane was proposed to be closely related to the homeostasis of $\left[\mathrm{Ca}^{2+}\right]_{\mathrm{i}}[7,8]$ with an unknown mechanism especially near the cell surface. 
Total internal reflection (TIR), an old concept of optics, has recently been applied to modern fluorescence microscopy and is becoming a new tool for investigating the dynamics of fluorescence-targeted molecules on or near the cell surface [9-12]. We have successfully set up a TIR fluorescence microscopy (TIRFM) system to investigate the movement of enhanced green fluorescent protein (EGFP)-targeted Rab3A protein [13] as well as mitochondrial functions [14] in rat pheochromocytoma PC12 cells. We further developed a dual-excitation TIRFM [15]. The dual-excitation lasers in this system can alternatively excite the surface fluorescent chromophores (eg, MitoTracker Green or rhod-2). By switching modes between epifluorescence (EPI) and TIR, we detected and compared mitochondrial functions between the two localizations (cytosolic and cell surface). In this study, both dynamics and changes in fluorescence intensity of components of interest, including mitochondrial motions and mitochondrial $\mathrm{Ca}^{2+}\left(\left[\mathrm{Ca}^{2+}\right]_{\mathrm{mt}}\right)$, in the cells were acquired and analyzed.

\section{Materials and methods}

\section{Cell culture and fluorescence labeling}

Rat adrenal pheochromocytoma cell line PC12 was obtained from the American Type Culture Collection (ATCC, Rockville, MD) and cultured as described previously $[13,16]$. Mitochondria and $\left[\mathrm{Ca}^{2+}\right]_{\mathrm{mt}}$ of $\mathrm{PC} 12$ cells were measured by MitoTracker Green and rhod-2 (Molecular Probes Inc, Eugene, OR), using the staining method as previously described [14].

\section{TIRFM}

The major component of our TIRFM system is a modified inverted microscope (IX-71, Olympus Optical Co., Ltd., Tokyo, Japan) with a high-numerical aperture objective $(\mathrm{NA}=1.45$, TIRFM, $60 \times$, Olympus $)$ and a EPI/TIR dual condenser (T.I.L.L. Photonics, Grafelfing, Germany). The TIRFM objective is controlled by a piezo $\mathrm{Z}$ driver (PI, Carlsruhe Germany). Two excitation light sources, $\mathrm{Ar}^{+}$and 532 lasers, were used to excite different fluorescent dyes at incident wavelengths of 488 and $532 \mathrm{~nm}$ separately into different emissions. With adapted dichroic mirrors, the switching excitation and emission spectra can be gathered by a high-performance cooled charge-coupled device (CCD; ORCA AG, Hamamatsu Photometrics, Hamamatsu, Japan). The full size of the CCD was used $(1344 \times 1024)$ without any binning to optimize the pixel $\left(6.45 \mu \mathrm{m}^{2}\right)$ to optical resolution $(\sim 200 \mathrm{~nm}$ at $520 \mathrm{~nm})$. Images were acquired by software SimplePCI 5.0 (Compix, Cranberry Township, PA), and some of them were further processed by the no-neighbors deconvolution algorithm, AutoDeblur 9.3 (AutoQuant Imaging, Inc. Troy, NY). Cells were treated with high- $\mathrm{K}^{+}$buffer as described previously [13].

\section{Online supplemental material}

Movie files available with the online version of this article, run at 12 frames/s, 10 times faster than the real sample of time-lapse images (1.2 frames/s).

\section{Results}

\section{Mitochondrial function assay by EPIFM/TIRFM}

Here we present a functional assay of mitochondria by using rhod-2, a fluorescent indicator of $\left[\mathrm{Ca}^{2+}\right]_{\mathrm{mt}}$ (Figures 1 and 2) under EPIFM/TIRFM. On our EPI/TIR dual switching platform, we can compare data from both images to investigate the role of mitochondrial localization within single cells. High- $\mathrm{K}^{+}$depolarization was used as a stimulation paradigm for observing the heterogeneity of mitochondria in different locations - in this case the cell surface and cytosol.

\section{Dynamics of mitochondria}

The behavior within living cells of mitochondria stained with MitoTracker Green was then further analyzed (Figure 2) under both EPI and TIR illumination. With proper time resolution (exposure time setting at $0.8 \mathrm{~s} /$ frame), serial timelapse images of mitochondria were acquired under both EPI (Figure 2, A) and TIR (Figure 2, B) illuminations and are shown superimposed in Figure 2, $C$ and $D$, and the movie clips available online (doi:10.1016/j.nano.2005.10.008). The motion of most mitochondria was suggested to be static under TIR excitation as shown in green color in Figure 2, $D$, or hovering under both illuminations as shown in Figure 2, $E$ within cytosolic fluid. In Figure 2, F, a rare vesicle-like mitochondrion moved quckly, as indicated by the arrow, under EPI excitation (shown in red). The range of mitochondrial displacement in living PC12 cells seldom exceeded $1 \mu \mathrm{m}$. As observed in the montage image display and online movie clips (doi:10.1016/j.nano.2005.10.008), the miniscule dynamic changes in the mitochondrial network could be seen; for example, in Figure 2, $D$ to $F$, the arrows and circle indicate the shape and localization of mitochondria and their dynamic changes. The behavior of mitochondria near the cell surface provides the real status of organelles within single living cells.

A few spontaneous movements of mitochondria similar to Figure 3, $F$ (red) can be also observed under TIR excitation. This unique movement is shown in Figure 4 and the online movie clips (doi:10.1016/j.nano.2005.10.008). The behavior of these mitochondria is similar to that of secretory vesicles. The rate of this vesicle-like movement of mitochondria is about $100 \mathrm{~nm} / \mathrm{s}$.

Images from confocal laser scanning (CLS) microscope-like optical section under TIR illumination

At the same laser incident angle that produced the TIR illumination, Figure 4 shows deconvoluted TIRFM images of MitoTracker Green-stained mitochondria within the same 

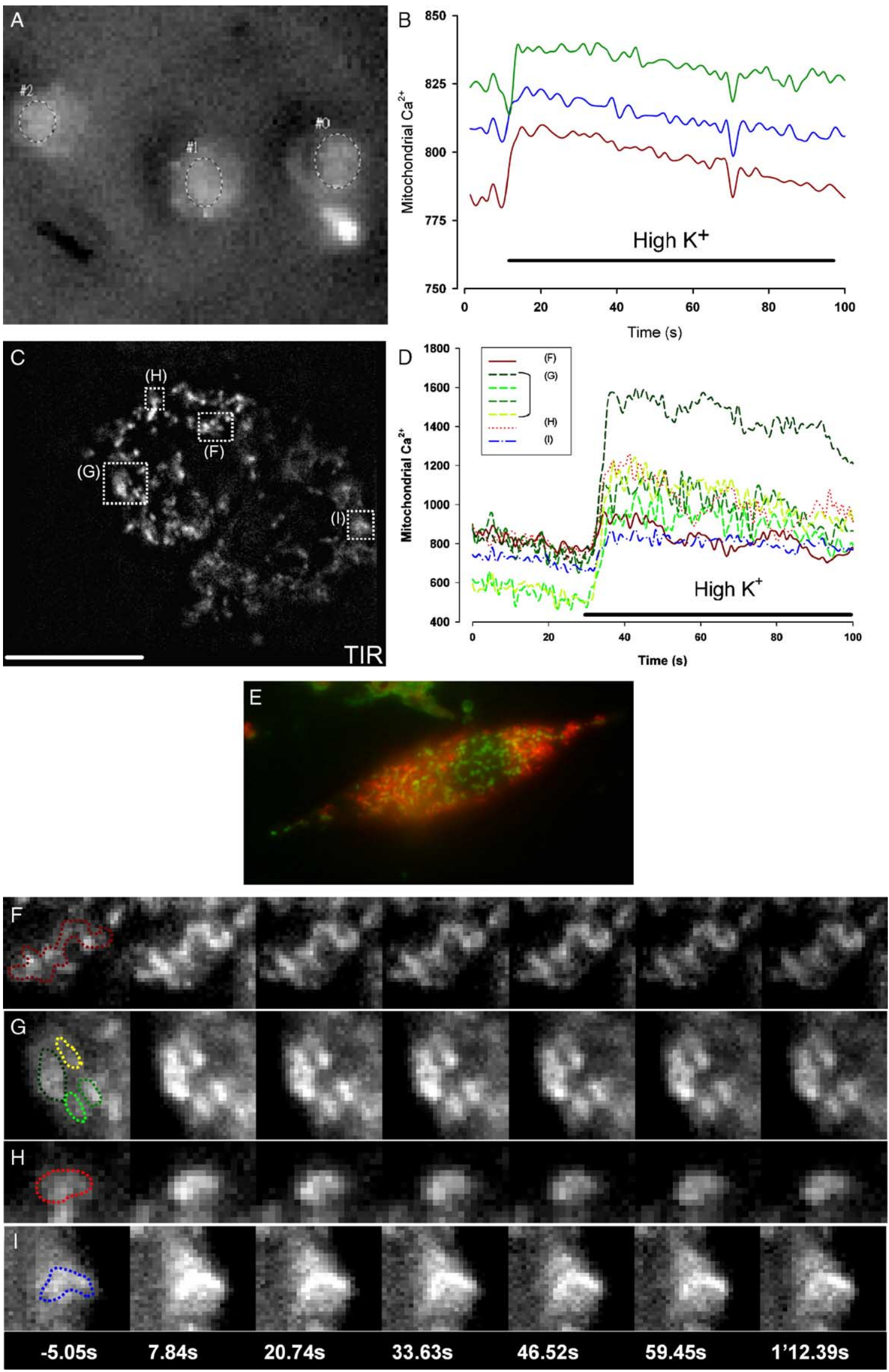

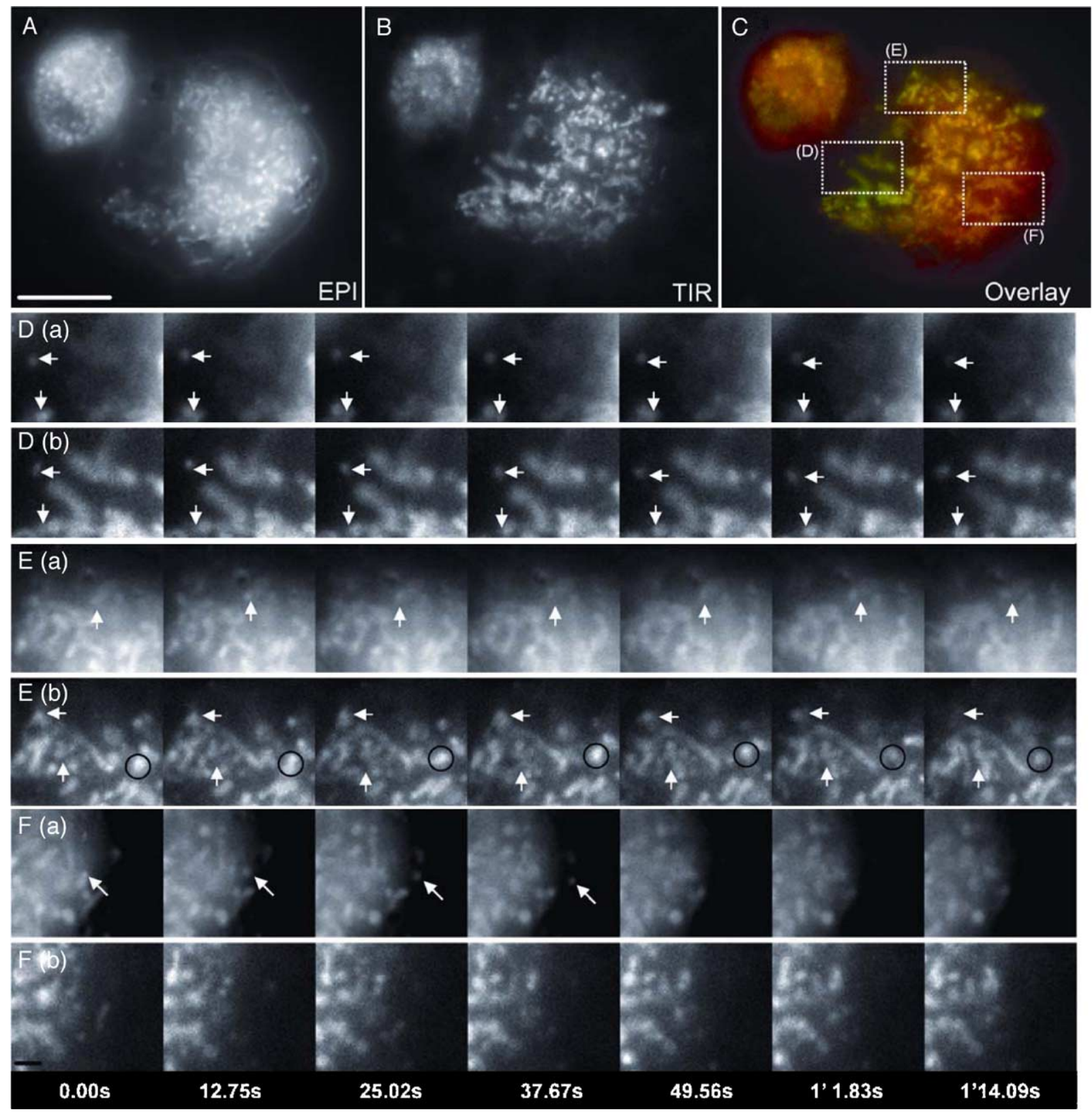

Fig 2. Image comparison and dynamics between EPI and TIR illumination mode acquisition of PC12 cells stained with MitoTracker Green. A, The fluorescence of mitochondria under EPI mode. B, The fluorescence of mitochondria under TIR mode. C, Overlay of EPI and TIR data within the same PC12 cells. D to F, As selected in the dash-lined boxes labeled D, E, and F in $\mathbf{C}$, overlay traces (every $\sim 12.7 \mathrm{~s} /$ frame as shown) of each time-lapse image in EPI (red) vs TIR (green) illumination are shown, respectively. Arrows and circle in D, E, and F indicate the movement of mitochondria under EPI or TIR mode. Scale bars in $\mathbf{A}$ and $\mathbf{F}=10$ and $1 \mu \mathrm{m}$, respectively.

PC12 cell acquired at different focal planes with steps of about $500 \mathrm{~nm}$ (from Figure 4, $A$ to $C$ ). The image taken at the focal plane nearest to the cell surface is shown in Figure $4, A$. Figure $4, D$ shows averaged intensity of 8 (closed circle), 11 (closed triangle), and 5 (closed diamond) selected MitoTracker Green-stained mitochondria at each section within the same PC12 cell selected from Figure $4, A, B$, and $C$, respectively. Figure $4, F$ and $G$ show another two sections of MitoTracker Green images within two PC12 cells. The reconstruction (overlay) image of these

Fig 1. Dynamics of mitochondrial $\mathrm{Ca}^{2+}$ located in cytosol and near the cell surface of single PC12 cells. A, Three single PC12 cells stained with rhod-2. B, Time-lapse data of $\left[\mathrm{Ca}^{2+}\right]_{\mathrm{mt}}$ viewed with EPIFM. C, Clear images of mitochondria stained with rhod-2 can be seen within a single cell. D, The time-lapse changes of $\left[\mathrm{Ca}^{2+}\right]_{\mathrm{mt}}$ via TIRFM of seven mitochondria in four dash-lined boxes of selected regions within two PC12 cells in $\mathbf{C}$. E, Merge images of EPI (red) and TIR (green) of mitochondria. $\mathbf{F}$ through $\mathbf{I}$, The movement of these seven mitochondria is shown every approximately $12.7 \mathrm{~s} /$ frame. Scale bar in $\mathbf{C}=10 \mu \mathrm{m}$. 

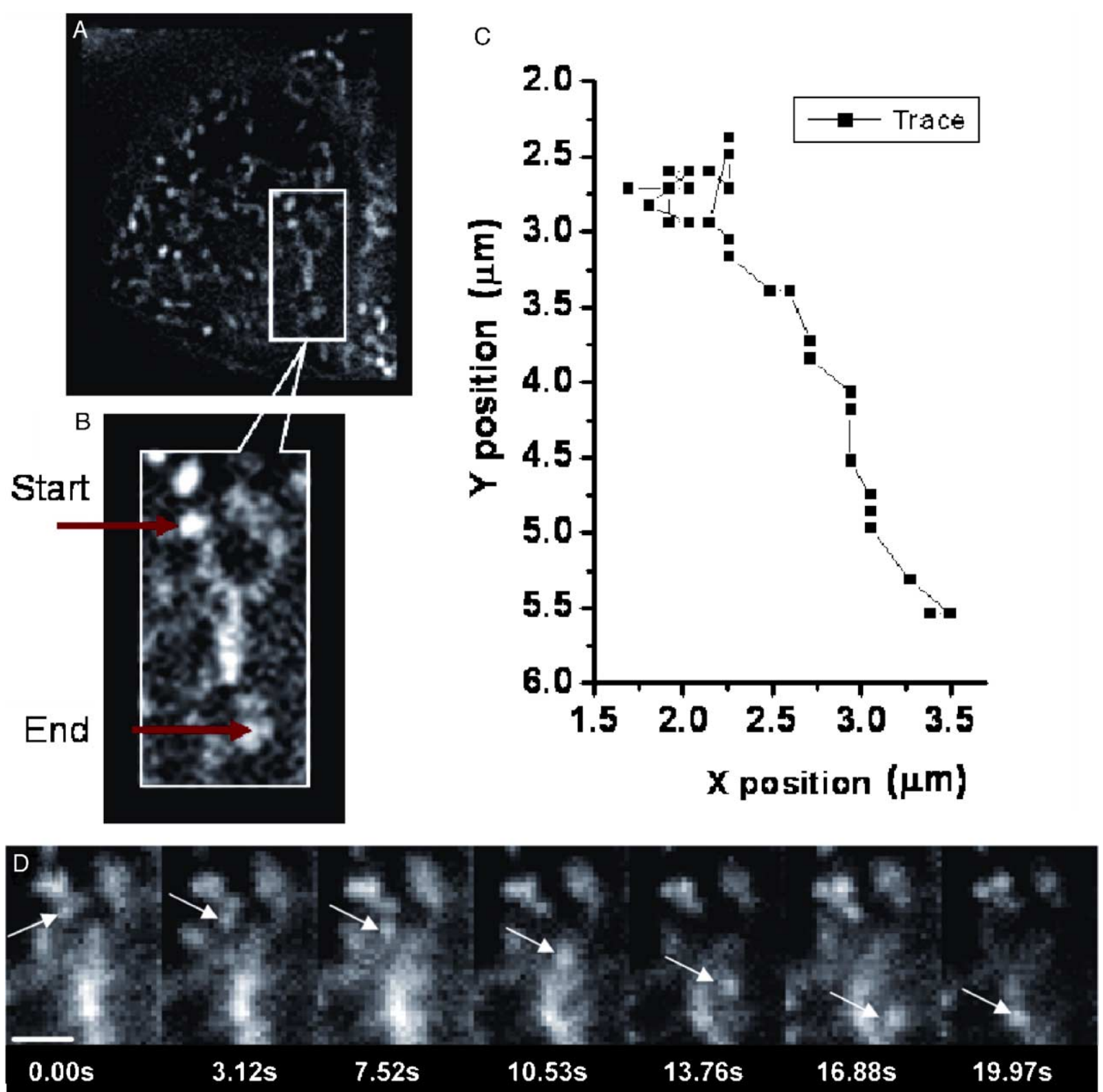

Fig 3. A vesicle-like mitochondrion near the cell surface was tracked within a single PC12 cell. A, Deconvoluted TIR image of a single PC12 cell stained with MitoTracker Green. B, Red arrow indicates the start and end of movement of this vesicle-like mitochondrion. C, Time-lapse motion trace and (D) images of this mitochondrion. The arrow in each trace of $\mathbf{D}$ indicates the location of this vesicle-like mitochondrion. The scale bar in $\mathbf{D}=1 \mu \mathrm{m}$.

sections (red image from section 1 in Figure 4, $F$ and green image from section 2 in Figure 4, $G$ ) is shown in Figure 4, $H$, and is similar to those made under CLS mode (data not shown).

\section{Discussion}

Use of rhod-2 to simultaneously record dynamics of $[\mathrm{Ca} 2+] \mathrm{mt}$ and mitochondrial movement

The $\left[\mathrm{Ca}^{2+}\right]_{\mathrm{mt}}$ indicator rhod-2 has been used in a variety of cell types, including neuroendocrine chromaffin cells [17] and nonexcitable T lymphocytes [18]. It has been suggested that the increase in $\left[\mathrm{Ca}^{2+}\right]_{\mathrm{mt}}$ plays an important role in activation of metabolism within mitochondria and also in the homeostasis of $\left[\mathrm{Ca}^{2+}\right]_{i}$ near the cell surface $[8,17]$. However, direct evidence proving that the effective mitochondria are located near the plasma membrane has not become available until recently [14]. In this study we used TIR illumination to acquire direct image data of rhod-2 near the cell surface. Time-lapse intensity data of $\left[\mathrm{Ca}^{2+}\right]_{\mathrm{mt}}$ were recorded and compared under TIR and EPI illumination. This comparison may provide direct evidence that the specific cell surface localization of mitochondria plays a critical role in regulating $\left[\mathrm{Ca}^{2+}\right]_{\mathrm{i}}$. More recently, techniques using GFP have been developed to monitor organelle calcium in situ by adding 

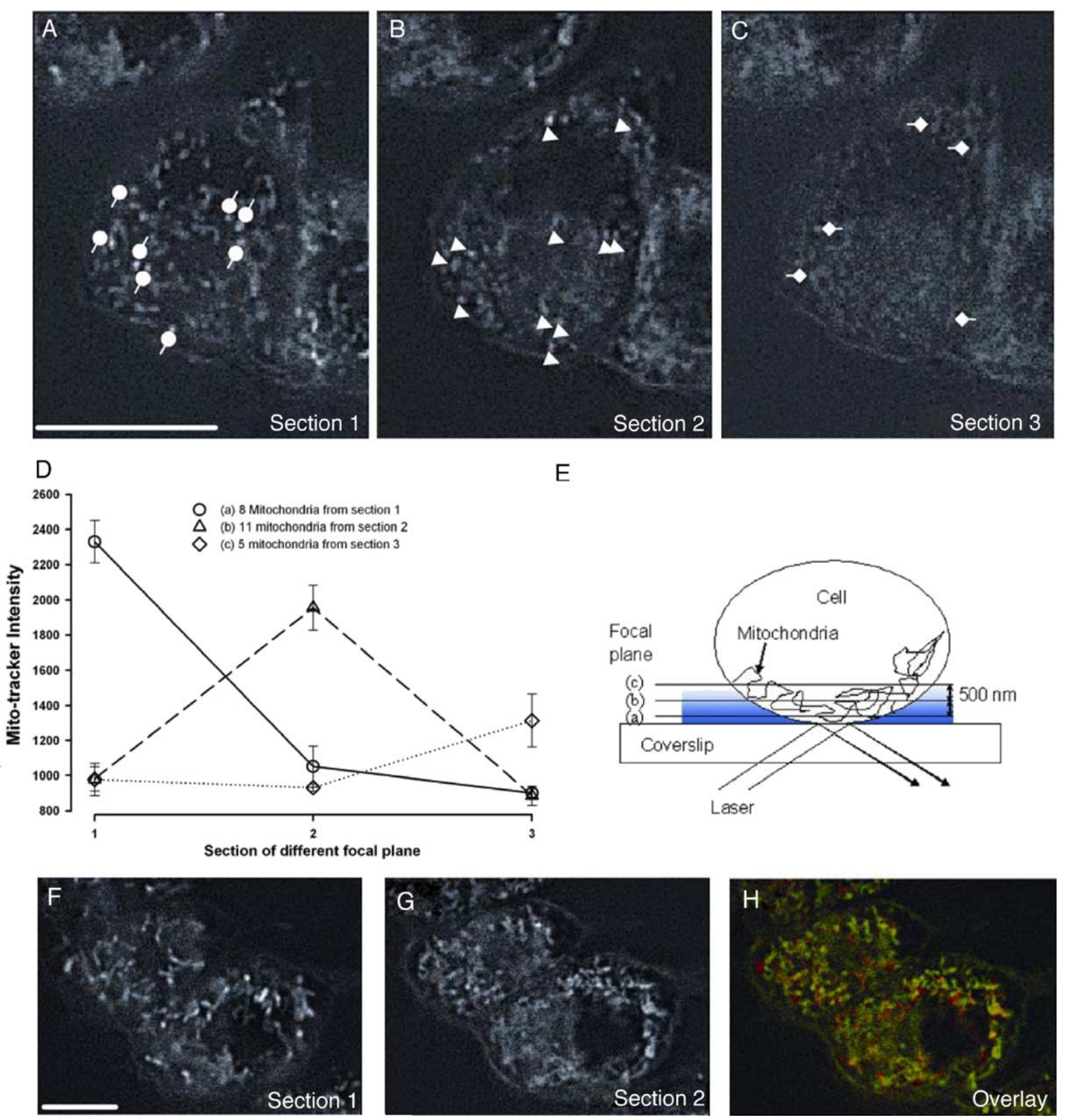

Fig 4. Optical sectioning test under TIRFM. Overlapped TIR fluorescent images of MitoTracker Green acquired at the same incident angle with different focal planes: 500-nm step from section 1 (A) to section 3 (C). In Section 1 (A), 2 (B), and 3 (C), 8 (closed circle), 11 (closed triangle), and 5 (closed diamond) mitochondria are selected, respectively. D, The averaged intensity of these selected mitochondria. $\mathbf{F}$ and $\mathbf{G}$, Another example, TIR images of two sections also with 500-nm steps. The intensity of $\mathbf{G}$ is readjusted to a better display. Images in $\mathbf{A}$ to $\mathbf{C}$ and $\mathbf{F}$ to $\mathbf{H}$ are all deconvoluted. $\mathbf{H}$, Overlapped image of Section 1 (red) and Section 2 (green). Scale bars in $\mathbf{A}$ and $\mathbf{F}=10 \mu \mathrm{m}$.

genes of signal peptides for specific organelles to $\mathrm{Ca}^{2+}$ sensors of a unique GFP, such as Cameleon or Pericam $[19,20]$. Such an alternative method would be an improvement for this research area, considering that the efficacy of rhod-2 is limited to $\left[\mathrm{Ca}^{2+}\right]_{\mathrm{mt}}$ measurement.

In this study most of the mitochondria near the cell surface and stained by MitoTracker Green and rhod-2 were seen to be static or floating within cytosolic fluid with some dynamic changes in their shape. However, we found one rare mitochondrion that seemed to fission from the mother mitochondrion and traveled for a long distance at the rate about $100 \mathrm{~nm} / \mathrm{s}$. The mechanism of this vesicle-like movement remains unknown. Previous reports suggested that some of the movement of mitochondria is related to cytoskeletal structures such as actin filaments or microtubules $[2,3]$.

\section{Image quality of fluorescence under different illuminations}

In a traditional fluorescent set-up, the image of rhod-2 under EPIFM using a xenon or mercury arc lamp as a light source was barely visible in detail (Figure 1). Under TIR illumination, both images of mitochondria and the $\left[\mathrm{Ca}^{2+}\right]_{\mathrm{mt}}$ of each single or network of mitochondria were revealed. Thus we can acquire the time-lapse traces of 
intensity changes as $\left[\mathrm{Ca}^{2+}\right]_{\mathrm{mt}}$ of each mitochondria from one cell, rather than only one selected datum from a whole cell. The resolution under TIR illumination was greatly enhanced because of the near-field excitation at both 488 $\mathrm{nm}$ (MitoTracker Green) and $543 \mathrm{~nm}$ (rhod-2) [9]. Several features of TIRFM should be noticed. First, it excites fluorescent molecules only near the plasma membrane $(\sim 100 \mathrm{~nm})$ as generated by the evanescent field. The fluorescent targets located more than $500 \mathrm{~nm}$ away from the cell surface could not be excited. Second, this nearfield illumination is weaker than the EPI light source. This near-field excitation greatly reduces the quenching effect that is the major problem in CLS microscopy. Images at a specific focal plane can be recorded through a pinhole in CLS microscopy, but laser illumination still excites certain parts of the sample at a different focal plane when scanning. Third, a fast-cooled CCD to improve time resolution can be used as an image detector rather than the generally slow-scanning photomultiplier tube needed with CLS microscopy. As seen in this study, the recording rate $(0.8 \mathrm{~s} /$ frame $)$ is ideally and approximately near the movement of mitochondria under TIRFM, and we are trying to optimize the rate to a higher speed while maintaining the detailed resolution. It is generally impossible to obtain the same image quality under a commercial CLS microscope at this rate or even faster.

\section{Optical sectioning function of TIRFM}

It has been reported that only small areas $(\sim 100 \mathrm{~nm})$ can be excited and that it is difficult to make optical sectioning under TIF illumination [9]. However, in a real sample this area could be larger than $200 \mathrm{~nm}$ [21]. The $e$-fold (space constant of fluorescence intensity declines) of PC12 cells proposed by Steyer and Almers [21] is about $640 \mathrm{~nm}$; this correlates with our result in this study (see below). The total length of the three sections shown in Figure 4 is about $1 \mu \mathrm{m}$. The fluorescence intensity became faint and blurred at such high positions because of the exponential decay of evanescent excitation. The overlays of TIR images were thus taken only from two sections (500 $\mathrm{nm}$ thickness) because of this nonlinear property of the evanescent wave. The reconstruction of these two TIRFM images is similar to that of CLS microscopy. Within an area less than $500 \mathrm{~nm}$ the optical sectioning can be done under TIRFM, and thus the three-dimensional (3D) or even four-dimensional (4D) images would be available under this set-up.

\section{Conclusions}

Both the time-lapse movement of mitochondria and changes in the amount of $\left[\mathrm{Ca}^{2+}\right]_{\mathrm{mt}}$ from single cells were detected and measured in this study. These detailed fluorescent images revealed the dynamics of mitochondria in living cells near the plasma membrane. In addition, the optical sectioning function of TIRFM allows for the acquisition of $3 \mathrm{D}$ or $4 \mathrm{D}$ live images with a much better time resolution than CLS microscopy under TIRFM.

\section{References}

[1] Fuler K, Arriaga EA. Advances in the analysis of single mitochondria. Curr Opin Biotechnol 2003;14:35-41.

[2] Scheffler IE. A century of mitochondrial research: achievements and perspectives. Mitochondrion 2001;1:3-31.

[3] Yaffe MP. The machinery of mitochondrial inheritance and behavior. Science 1999;283:1493-7.

[4] Shaw JM, Nunnari J. Mitochondrial dynamics and division in budding yeast. Trends Cell Biol 2002;12:178-84.

[5] Adrain C, Creagh EM, Martin SJ. Apoptosis-associated release of Smac/DIABLO from mitochondria requires active caspases and is blocked by Bcl-2. EMBO J 2001;20:6627-36.

[6] Carafoli E. Historical review: mitochondria and calcium: ups and down of an unusual relationship. Trends Biochem Sci 2003;4:175 -81.

[7] Rizzuto R, Pinton P, Carrington W, Fay FS, Fogarty KE, Lifshitz LM, et al. Close contacts with endoplasmic reticulum as determinants of mitochondrial $\mathrm{Ca}^{2+}$ responses. Science 1998;280:1763-6.

[8] Yang DM, Kao LS. Relative contribution of the $\mathrm{Na}^{+} / \mathrm{Ca}^{2+}$ exchanger, mitochondria and endoplasmic reticulum in the regulation of cytosolic $\mathrm{Ca}^{2+}$ and catecholamine secretion of bovine adrenal chromaffin cells. J Neurochem 2001;76:210-6.

[9] Axelord D. Total internal reflection fluorescence microscopy. Methods Cell Biol 1989;30:245-70.

[10] Axelord D. Total internal reflection fluorescence microscopy in cell biology. Traffic 2001;2:764-74.

[11] Stephens DJ, Allan VJ. Light microscopy techniques for living cell imaging. Science 2003;300:82-6.

[12] Toomre D, Manstain DJ. Lighting up the cell surface with evanescent wave microscopy. Trends Cell Biol 2001;11:298-303.

[13] Yang DM, Huang CC, Lin HY, Tsai DP, Kao LS, Chi CW, et al. Tracking of secretory vesicles of PC12 cells using total internal reflection fluorescence microscopy. J Microsc 2003;209:223-7.

[14] Yang DM, Lin CC, Lin HY, Huang CC, Tsai DP, Chi CW, et al. Dynamics of mitochondria and mitochondrial $\mathrm{Ca}^{2+}$ near the plasma membrane of PC12 cells: a study by multimode microscopy. Ann N Y Acad Sci 2005;1042:163-7.

[15] Merrifield CJ, Feldman ME, Wan L, Almers W. Imaging actin and dynamin recruitment during invagination of single clathrin-coated pits. Nat Cell Biol 2002;4:691 - 8 .

[16] Huang CM, Kao LS. Nerve growth factor, epidermal growth factor, and insulin differentially potentiate ATP-induced $\left[\mathrm{Ca}^{2+}\right] \mathrm{i}$ rise and dopamine secretion in PC12 cells. J Neurochem 1996;66: $124-30$.

[17] Babcock DF, Herrington J, Goodwin PC, Park YB, Hille B. Mitochondrial participation in the intracellular $\mathrm{Ca}^{2+}$ network. J Cell Biol 1997; 136:833-44.

[18] Hoth M, Fanger CM, Lewis RS. Mitochondrial regulation of storeoperated calcium signaling in T lymphocytes. J Cell Biol 1997; 137:633-48.

[19] Miyawaki A, Griesbeck O, Heim R, Tsien RY. Dynamics and quantitative $\mathrm{Ca}^{2+}$ measurements using improved cameleons. Proc Natl Acad Sci U S A 1999;96:2135-40.

[20] Nagai T, Sawano A, Park ES, Miyawaki A. Circularly permuted green fluorescent proteins engineered to sense $\mathrm{Ca}^{2+}$. Proc Natl Acad Sci U S A 2001;98:3197-202.

[21] Steyer JA, Almers W. Tracking single secretory granules in live chromaffin cells by evanescent-field fluorescence microscopy. J Biophys 1999;76:2262 - 71 . 